\begin{tabular}{|c|c|c|}
\hline UFJ/UFG & $\begin{array}{c}\text { GEOAMBIENTE ON-LINE } \\
\text { Revista Eletrônica do Curso de Geografia } \\
\text { Graduação e Pós-Graduação } \\
\text { Htpp://www.revistas.ufg.br/geoambiente } \\
\text { Jataí-GO | n 37 | Mai-Agos/2020 }\end{array}$ & $\begin{array}{l}\text { EGEO } \\
\text { ISSN } 1679-9860\end{array}$ \\
\hline
\end{tabular}

\title{
MODELOS CHUVA-VAZÃO: USO DE TÉCNICAS DE APRENDIZAGEM DE MÁQUINAS PARA CALIBRAÇÃO DE MODELOS EM UMA PEQUENA BACIA HIDROGRÁFICA
}

\author{
Thiago Boeno Patricio Luiz ${ }^{1}$, Thomas Schröder ${ }^{2}$
}

(1 - Universidade Federal de Santa Maria - UFSM, e-mail: thiagoboeno@hotmail.com, https://orcid.org/0000-0002-7054-1780, 2 - Fundação Municipal de Meio Ambiente de Biguaçu FAMABI, e-mail: thomaschroder@gmail.com, http://orcid.org/0000-0001-5550-1935)

Resumo: Estimativas de vazões em bacias hidrográficas baseadas em dados de precipitação pluviométrica são extremamente importantes para atividades relacionadas à gestão dos recursos hídricos. A elaboração de cenários de disponibilidade hídrica com boa precisão pode contribuir com os processos de planejamento dos recursos ambientais e evitar possíveis conflitos pelo uso da água. Este trabalho utilizou estruturas baseadas em aprendizagem de máquinas (Machine Learning) para calibrar dois modelos de chuva-vazão em escala diária na Bacia Hidrográfica do Arroio Grande no leste do Rio Grande do Sul. Foram empregados métodos de Redes Neurais Artificiais (RNA) e Gradient Boosting Machine (GBM) com a técnica bootstrap de reamostragem. O objetivo deste trabalho foi avaliar a capacidade dessas técnicas para modelar a série histórica de vazão, considerando-se a influência de dois pluviômetros localizados próximos à estação fluviométrica. A performance das técnicas utilizadas foi verificada por meio do coeficiente de determinação $\left(R^{2}\right)$, que atingiu 0,93 para o algoritmo de redes neurais e de 0,99 para o algoritmo de boosting, bem como pelos baixos valores do desvio absoluto. Através dos gráficos de resíduos foi possível observar o bom desempenho de calibração alcançado na aplicação dessas técnicas, onde a técnica GBM apresentou-se levemente superior à de RNA.

Palavras-chave: Hidrologia. Vazão. Redes Neurais Artificiais. Gradient Boosting Machine.

Artigo recebido para publicação em 07 de Abril de 2020

Artigo aprovado para publicação em 16 de Julho de 2020 


\begin{tabular}{|c|c|c|}
\hline UFJ/UFG & $\begin{array}{c}\text { GEOAMBIENTE ON-LINE } \\
\text { Revista Eletrônica do Curso de Geografia } \\
\text { Graduação e Pós-Graduação } \\
\text { Htpp://www.revistas.ufg.br/geoambiente } \\
\text { Jataí-GO | n 37 | Mai-Agos/2020 }\end{array}$ & $\begin{array}{l}\text { :GEO } \\
\text { :̇AMBIENTE } \\
\text { ISSN } 1679-9860\end{array}$ \\
\hline
\end{tabular}

\title{
RAINFALL RUNOFF MODELS: USE OF MACHINE LEARNING TECHNIQUES FOR MODEL CALIBRATION IN A SMALL WATERSHED
}

\begin{abstract}
Flow rate estimates in river basins based on rainfall data are extremely important for activities related to management of water resources. The elaboration of water availability scenarios with good precision can contribute to the planning of environmental resources and avoid possible conflicts over the use of water. This paper used structures based on machine learning to calibrate two rain-flow models on a daily scale at Arroio Grande Hydrographic Basin in eastern of Rio Grande do Sul. Artificial Neural Network (ANN) and Gradient Boosting Machine (GBM) methods were used with the resampling bootstrap. The objective of this paper was to evaluate the capacity of these techniques to model the historical flow series, considering the influence of two rain gauges located close to the river-gauge station. The performance of the techniques used was verified by the determination coefficient $\left(\mathrm{R}^{2}\right)$, which was 0,93 for the neural network algorithm and 0,99 for the boosting algorithm, as well as the low values of the absolute deviation. Through the graphs of residues, it was possible to observe the good calibration performance achieved in the application of these techniques, where the GBM technique was slightly superior to that of ANN.
\end{abstract}

Keywords: Hydrology. Flow rate. Artificial Neural Network. Gradient Boosting Machine.

\section{MODELOS LLUVIA-CAUDAL: USO DE TÉCNICAS DE APRENDIZAJE DE MÁQUINAS PARA CALIBRAR MODELOS EN UNA PEQUEÑA CUENCA HIDROGRÁFICA}

Resumen: Las estimaciones de caudales en las cuencas hidrográficas basadas en datos de lluvia son extremadamente importantes para las actividades relacionadas con la gestión de los recursos hídricos. La elaboración de escenarios de disponibilidad de agua con buena precisión puede contribuir a los procesos de planificación de los recursos ambientales y evitar posibles conflictos por el uso del agua. Este trabajo utilizó estructuras basadas en el aprendizaje automático (Machine Learning) para calibrar dos modelos lluvia-caudal a paso diario en la cuenca del río Arroio Grande, en el este de Rio Grande do Sul. Se utilizaron Redes Neuronales Artificiales (RNA) y los métodos de Gradient Boosting Machine (GBM) con técnica de bootstrap. El objetivo de este trabajo fue 


\begin{tabular}{|c|c|c|}
\hline UFJ/UFG & $\begin{array}{c}\text { GEOAMBIENTE ON-LINE } \\
\text { Revista Eletrônica do Curso de Geografia } \\
\text { Graduação e Pós-Graduação } \\
\text { Htpp://www.revistas.ufg.br/geoambiente } \\
\text { Jataí-GO | n 37 | Mai-Agos/2020 }\end{array}$ & $\begin{array}{l}\text { :GEO } \\
\text { :̇AMBIENTE } \\
\text { ISSN } 1679-9860\end{array}$ \\
\hline
\end{tabular}

evaluar la capacidad de estas técnicas para modelar la serie histórica de caudales, considerando la influencia de dos pluviómetros ubicados cerca de la estación de aforo. El rendimiento de las técnicas utilizadas se verificó mediante el coeficiente de determinación $\left(\mathrm{R}^{2}\right)$, que alcanzó 0,93 para el algoritmo de red neuronal y 0,99 para el algoritmo de boosting, así como los valores bajos de la desviación absoluta. Mediante los gráficos residuales fue posible observar el desempeño de calibración logrado en la aplicación de estas técnicas, donde la técnica GBM fue ligeramente superior a la del RNA.

Palabras clave: Hidrología. Caudales. Redes Neuronales Artificiales. Gradient Boosting Machine.

\section{Introdução}

A previsão de dados hidrológicos em bacias hidrográficas constituí uma ferramenta de alta importância para situações que demandam o planejamento e a gestão dos recursos hídricos. Modelos de chuva-vazão são utilizados para realizar simulações hidrológicas e para fornecer subsídios para a regionalização de vazões em bacias hidrográficas, uma vez que permitem a predição do regime fluvial.

Para atingir tal fim, existem algumas abordagens que podem ser empregadas como o uso de modelos físicos ou determinísticos, modelos estatísticos ou estocásticos e modelos baseados em algoritmos de aprendizagem de máquinas (Machine Learning). Modelos de aprendizagem se adaptam ao conjunto amostral monitorado, constituindo-se como estruturas empíricas guiadas pelos dados. Assim, essas abordagens devem sempre levar em consideração a disponibilidade de dados para simulação e o grau de precisão que cada técnica apresenta ao reproduzir o fenômeno estudado.

Estruturas autorregressivas em modelos de séries temporais utilizam observações anteriores como entrada para uma equação de regressão, a fim de prever o valor da próxima observação. É uma ideia simples, mas que pode resultar em previsões mais aprimoradas sobre uma série de problemas, principalmente quando combinadas com técnicas de aprendizagem computacional.

Nessa perspectiva, o uso de técnicas baseadas em aprendizagem de máquinas tem se intensificado cada vez mais, devido aos bons resultados encontrados em distintos segmentos da hidrologia como Snelder et al. (2009), Maier et al. (2010), Souza e Souza (2010), He et al. (2014), 


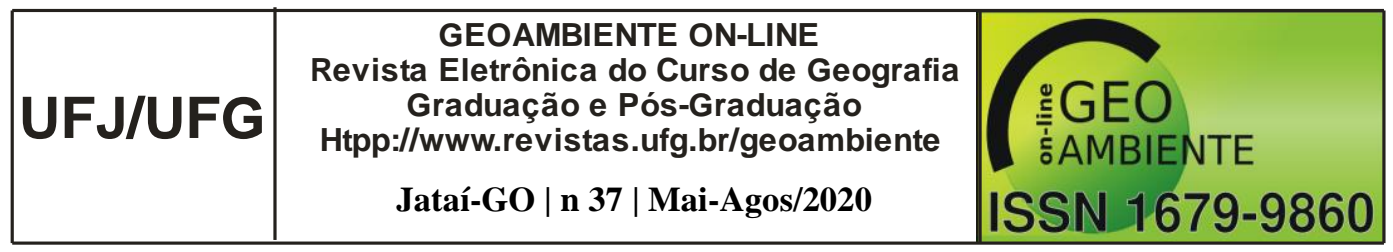

Li et al., (2018), Mosavi et al. (2018), Worland et al. (2018), Senent-Aparicio et al. (2019), entre outros.

Dentre os métodos de aprendizagem de máquina, destacam-se os modelos baseados em Redes Neurais Artificiais (do inglês Artificial Neural Networks), muito utilizados em diversos estudos e amplamente aceitos no meio científico. As Redes Neurais têm demonstrado resultados significativos pois são muito úteis para se modelar sistemas não-lineares complexos com grande quantidade de dados (SOUZA E SOUZA, 2010).

Os algoritmos de Gradient Boosting (FRIEDMAN, 2001) é uma família de técnicas de aprendizagem de máquina que, recentemente, mostraram-se muito positivas em uma série de aplicações práticas (FONSECA et al., 2019; LIAO et al., 2020; NI et al., 2020). Os termos boosting (impulso) e gradient (gradiente) podem ser interpretados a partir da idealização de um algoritmo de otimização que opera sob uma ou várias funções de perda.

A técnica de Gradient Boosting Machine (GBM) é uma combinação de diferentes modelos para produzir predições, uma vez que usa dois algoritmos: árvores de regressão e boosting, este último que constrói e combina os modelos. As árvores de regressão dividem o espaço de predição em retângulos com base nas variáveis independentes, ajustando uma constante em cada subespaço (FRIEDMAN, 2001). A variável independente e os pontos de divisão binários são escolhidas de forma a minimizar a entropia do modelo. A árvore de regressão cresce com a adição de novas variáveis ou novos pontos de divisão até que um critério seja alcançado.

O aprimoramento dos modelos hidrológicos baseados em aprendizagem de máquinas vem permitindo aumentar a performance preditiva em diversos casos a partir do desenvolvimento de diferentes modelos de predição. Este trabalho visa comparar uma técnica de Rede Neural Artificial do tipo feedforward com o algoritmo de Gradient Boosting Machine na calibração de modelos autorregressivos de chuva-vazão na Bacia Hidrográfica do Arroio Grande. Assim, o estudo busca contribuir com o entendimento de técnicas que sejam capazes de minimizar as incertezas para melhor realizar estudos dos regimes fluviais de pequenas bacias e com dados escassos.

\section{Material Métodos}

\subsection{Localização e descrição da área}




\begin{tabular}{|l|c|c|}
\hline UFJ/UFG & $\begin{array}{c}\text { GEOAMBIENTE ON-LINE } \\
\text { Revista Eletrônica do Curso de Geografia } \\
\text { Graduação e Pós-Graduação } \\
\text { Htpp://www.revistas.ufg.br/geoambiente } \\
\text { Jataí-Go | n 37 | Mai-Agos/2020 }\end{array}$ & $\begin{array}{l}\text { ISEO } \\
\text { ISTM 1679-9860 }\end{array}$ \\
\hline
\end{tabular}

A Bacia Hidrográfica do Arroio Grande (BHAG) localiza-se majoritariamente no município de Glorinha, no Estado do Rio Grande do Sul (Figura 1), fazendo parte da Bacia Hidrográfica do Rio Gravataí na região hidrográfica do Guaíba. A BHAG faz parte das cabeceiras do rio Gravataí e deságua em uma área formadora de complexos de banhados conhecida como Banhado Grande, a qual é uma Área de Proteção Ambiental (APA) criada pelo Decreto Estadual $\mathrm{n}^{\circ} 38.971$ (RIO GRANDE DO SUL, 1998).

Figura 1 - Localização da Bacia Hidrográfica do Arroio Grande com destaque ao Banhado Grande e estações utilizadas localizadas no leste do Estado do Rio Grande do Sul.

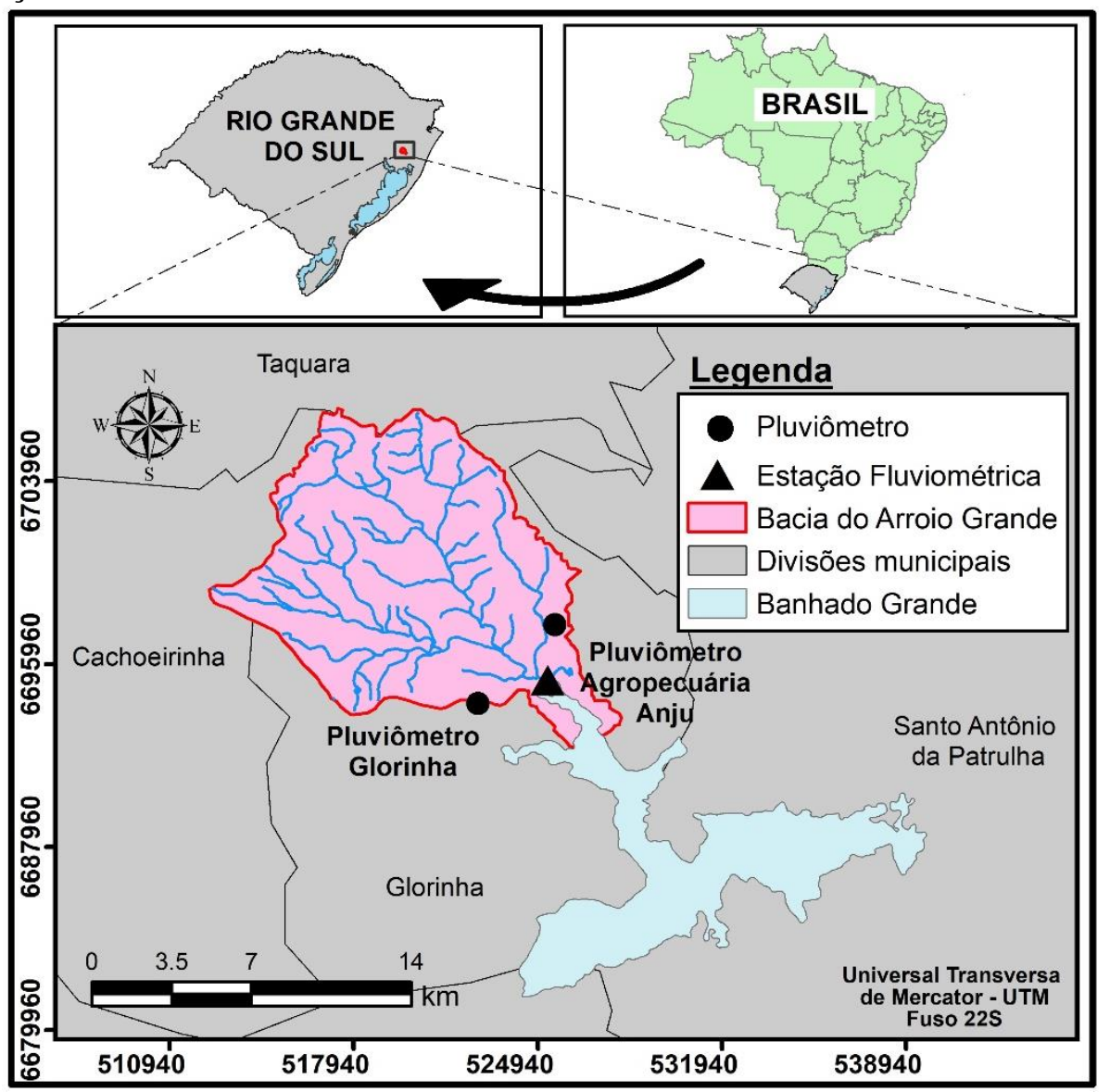

Organização: Autores.

Essa APA é um importante ecossistema natural da área de estudo, localizada na intersecção dos biomas Pampa e Mata Atlântica, sendo refúgio de vida silvestre onde predominam restingas, banhados e as nascentes do rio Gravataí (SEMA, 2020). Atualmente, a APA possui em seu território áreas urbanas e atividades agropastoris, com predominância de orizicultura irrigada. 


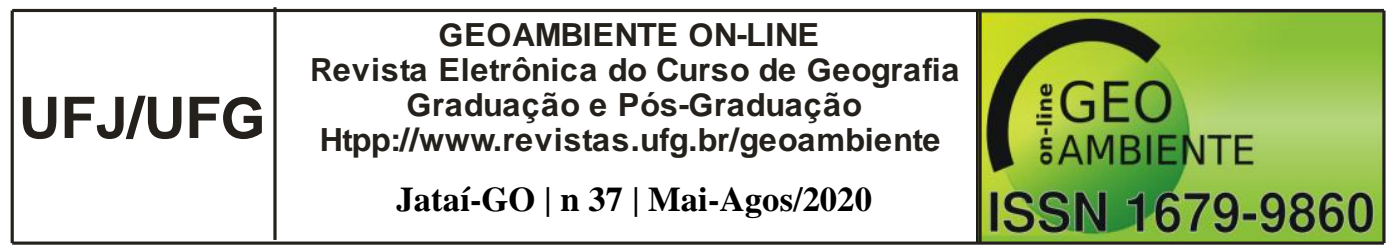

Essas atividades causaram ao longo dos anos uma série de impactos ambientais nessas áreas úmidas (BELLOLI; GUASSELLI, 2018), com implicâncias diretas no regime hídrico do rio Gravataí.

Os dados de vazão e de precipitação foram adquiridos junto ao Sistema de Informações Hidrológicas HIDROWEB (ANA, 2019) mantida pela Agência Nacional de Águas (ANA). O conjunto de dados utilizado foi de 01 de setembro de 2012 a 31 de dezembro de 2015 , totalizando 1217 medições de vazão e de precipitação sem a ocorrência de falhas no período. A estação fluviométrica utilizada foi a do Passo Grande (estação 87398800), operada pela Companhia de Pesquisa em Recursos Minerais (CPRM) localizada às margens da estrada RS 030 e captando uma área de drenagem de aproximadamente $120 \mathrm{~km}^{2}$.

Os pluviômetros utilizados denominados Glorinha (pluviômetro 02950016) e Agropecuária Anju (pluviômetro 02950059) são operados pela ANA, estando distantes cerca de 5,3 km e 6,2 km da estação fluviométrica respectivamente (Figura 1). A avaliação referente à qualidade dos dados foi feita por inspeção visual, onde foi analisada a consistência a partir de períodos em que não houveram falhas nas medições.

\subsection{Caracterização e Pré-processamento dos Dados}

Na Figura 2 apresenta-se o Hidrograma diário com o registro de vazões da $B H A G$ em m³/s e suas principais características descritivas. As operações de estatística bem como os ajustes dos modelos foram realizadas por meio da linguagem de programação R (R CORE TEAM, 2020).

As vazões registradas para a BHAG no período selecionado para o estudo obtiveram uma vazão mínima de $0,56 \mathrm{~m}^{3} / \mathrm{s}$ e uma vazão máxima de $20,1 \mathrm{~m}^{3} / \mathrm{s}$, totalizando uma amplitude de 19,54 $\mathrm{m}^{3} / \mathrm{s}$ e um Coeficiente de Variação (CV) de $115 \%$. O cálculo da Q95 ficou na ordem de 0,82 m³/s, ou seja, apresentou uma vazão mínima de $0,82 \mathrm{~m} 3 / \mathrm{s}$ em $95 \%$ do tempo estudado.

Os dados de precipitação nos pluviômetros utilizados obtiveram máximas diárias de até 111 milímetros no pluviômetro Glorinha e atingiu 118 milímetros no pluviômetro Agropecuária Anju. Em geral, os dados diários dos dois pluviômetros obtiveram uma forte tendência de correlação linear, uma vez que estão relativamente próximos um do outro, atingindo um coeficiente de correlação de 0,70. A precipitação anual acumulada registrada nos anos de 2013 a 2015 tiveram médias na ordem de 1657 milímetros no pluviômetro de Glorinha e 1785 milímetros no 


\begin{tabular}{|c|c|c|}
\hline UFJ/UFG & $\begin{array}{c}\text { GEOAMBIENTE ON-LINE } \\
\text { Revista Eletrônica do Curso de Geografia } \\
\text { Graduação e Pós-Graduação } \\
\text { Htpp://www.revistas.ufg.br/geoambiente } \\
\text { Jataí-GO | n 37 | Mai-Agos/2020 }\end{array}$ & $\begin{array}{l}\text { :GEO } \\
\text { :AMBIENTE } \\
\text { ISSN } 1679-9860\end{array}$ \\
\hline
\end{tabular}

pluviômetro da Agropecuária Anju, estando dentro das médias anuais naturais da região segundo Alvares et al. (2013).

Figura 2 - Hidrograma das vazões diárias registradas na estação fluviométrica do Passo Grande com as informações da estatística descritiva básica.

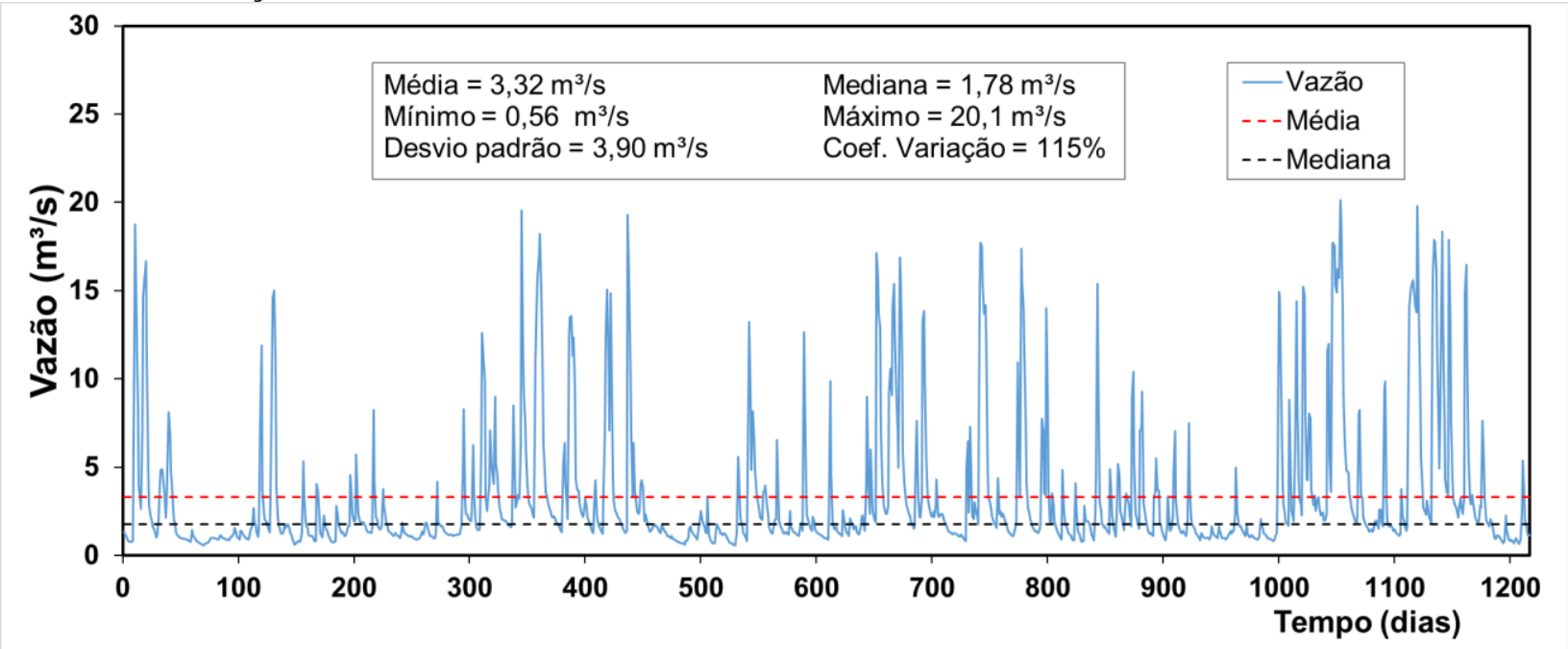

Fonte: Próprio Autores.

Essas séries históricas de dados foram pré-processadas e avaliadas a partir de sua autocorrelação e através da correlação cruzada entre elas. O objetivo destes procedimentos foi de entender as relações de interdependência entre as observações das séries temporais para propor estruturas correlacionadas para calibração de um modelo autorregressivo.

\subsection{Ajuste do modelo a partir de Redes Neurais Artificiais}

O ajuste do modelo por RNA foi feito utilizando apenas uma camada escondida com o algoritmo do tipo feedforward com a chuva do primeiro e do segundo pluviômetro. Os dados foram centrados e padronizados antes da calibração. Neste algoritmo, as conexões entre as unidades (neurons) não formam ciclos. A função de ativação utilizada em cada unidade foi a função logística. A função de entropia (ou função de perda) utilizada para a avaliação do ajuste foi a dos mínimos quadrados, uma rede neural foi considerada ajustada quando o otimizador foi incapaz de reduzir este critério em magnitudes menores que $10^{-8}$. 


\begin{tabular}{|c|c|c|}
\hline UFJ/UFG & $\begin{array}{c}\text { GEOAMBIENTE ON-LINE } \\
\text { Revista Eletrônica do Curso de Geografia } \\
\text { Graduação e Pós-Graduação } \\
\text { Htpp://www.revistas.ufg.br/geoambiente } \\
\text { Jataí-GO | n 37 | Mai-Agos/2020 }\end{array}$ & $\begin{array}{l}\text { :GEO } \\
\text { :AMBIENTE } \\
\text { ISSN } 1679-9860\end{array}$ \\
\hline
\end{tabular}

O grau de ajuste de uma feedforward depende do número de unidades e da taxa de aprendizado. Como forma de identificar a combinação destes hiperparâmetros que produz o melhor ajuste foi construída uma grade de modelos, com o número de unidades variando de 5 a 10 e a taxa de aprendizado variando de 0,0001 até 0,1 em passos de 0,0002, estabelecendo para tanto um limite de 10000 interações para convergência do algoritmo. Desta forma, foram testadas um total de 300 arquiteturas de redes neurais usando o algoritmo disponível no pacote nnet (RIPLEY; VENABLES, 2020).

\subsection{Ajuste do modelo a partir de Gradient Boosting Machine}

A qualidade do ajuste de um modelo que usa GBM depende, geralmente, do número de árvores de regressão utilizados, do tamanho destas, da taxa de aprendizado e da profundidade de interação entre as variáveis dependentes. O tamanho das árvores foi controlado pelo número mínimo de conjuntos observados em uma divisão terminal de cada árvore (por vezes chamada de folha). Este número foi limitado a 10 observações de acordo com a literatura (RIDGEWAY, 2019).

Os demais hiperparâmetros do modelo foram testados de forma sistemática através de uma grade de predição. O número de árvores utilizado variou de 200 a 2000 em passos de 600, a taxa de aprendizado foi testada em taxas de 0,0001 até 0,5 em passos de 0,03 e a profundidade de interação entre as variáveis independentes variou de 3 a 7 em passos unitários. Foram testadas 340 diferentes combinações de hiperparâmetros utilizando o pacote gbm (RIDGEWAY, 2019).

\subsection{Ajuste dos modelos de Aprendizado de Máquina}

A forma ideal de avaliar o ajuste de um dado modelo seria através da obtenção de novas amostras da população. Uma prática recorrente consiste na divisão dos dados disponíveis em dois conjuntos de dados, um para ajuste (comumente chamado de conjunto de treinamento) e um para validação (também chamado de conjunto de teste) em proporções variadas da totalidade dos dados. Além dos problemas inerentes a uma escolha aleatória do conjunto de dados para teste (WENGER; OLDEN, 2012) esta abordagem corresponde a um desperdício de informação por não utilizar todos os dados para o ajuste do modelo, causando uma superestimativa do erro do conjunto de dados de 


\begin{tabular}{|c|c|c|}
\hline UFJ/UFG & $\begin{array}{c}\text { GEOAMBIENTE ON-LINE } \\
\text { Revista Eletrônica do Curso de Geografia } \\
\text { Graduação e Pós-Graduação } \\
\text { Htpp://www.revistas.ufg.br/geoambiente } \\
\text { Jataí-GO | n 37 | Mai-Agos/2020 }\end{array}$ & $\begin{array}{l}\text { :GEO } \\
\text { :AMBIENTE } \\
\text { ISSN } 1679-9860\end{array}$ \\
\hline
\end{tabular}

teste (KOHAVI, 1995; HASTIE et al., 2009). Para superar estas limitações foram utilizados métodos de reamostragem.

No presente estudo foi utilizado o método de bootstrap, que tem demonstrado bons resultados em diversos estudos (BORRA; DI CIACCIO, 2010), com destaque em usos na hidrologia (ZHANG et al., 2017; BARZEGAR et al., 2019; LI et al., 2019), entre outros. No método de bootstrap o conjunto de dados é reamostrado com reposição até a formação de um conjunto de dados artificial de mesmo tamanho do conjunto de dados original.

O modelo é então ajustado a este conjunto artificial e seu ajuste avaliado com base nos dados deixados de fora do conjunto artificial. No entanto, este método pode superestimar o erro de um dado modelo, para evitar este problema foi utilizado o método que condiciona o número de amostras do conjunto de dados originais a 63,2\% destes (EFRON; TIBSHIRANI, 1997). Este processo foi repetido 25 vezes (PATTENGALE et al., 2010) para cada modelo e os melhores resultados foram combinados de forma a gerar somente um modelo preditivo.

Para o ajuste dos diferentes métodos, utilizou-se o pacote Caret (Classification and Regression Training) (KUHN, 2019) a fim de simplificar as nuances sintáticas de cada técnica de machine learning e facilitar o processo de treinamento dos modelos

\subsection{Avaliação dos modelos calibrados}

Os modelos foram testados através de erros (entropias) de ordem quadrática e aritmética. Como estatística para representação dos erros de ordem quadrática foi utilizado o coeficiente de determinação $\left(\mathrm{R}^{2}\right)$ (equação 1) e, para representação dos erros de ordem aritmética, foi utilizado o Desvio Absoluto, ou seja, o somatório da diferença absoluta (módulo) entre os valores observados e os estimados (equação 2). Uma análise mais detalhada dos resíduos foi obtida com a análise gráfica da dispersão dos erros em função da variável dependente estimada e em função de todas variáveis independentes para um dado tempo t.

$$
\begin{aligned}
& R^{2}=\frac{\left[\sum_{i=1}^{k}\left(o_{i}-\bar{o}_{i}\right) \cdot\left(t_{i}-\bar{t}_{i}\right)\right]^{2}}{\sum_{i=1}^{k}\left(o_{i}-\bar{o}_{i}\right)^{2} \cdot \sum_{i=1}^{k}\left(t_{i}-\bar{t}_{i}\right)^{2}} \\
& \text { Desvio Absoluto }=\sum_{i=1}^{k}\left|o_{i}-t_{i}\right|
\end{aligned}
$$




\begin{tabular}{|c|c|c|}
\hline UFJ/UFG & $\begin{array}{c}\text { GEOAMBIENTE ON-LINE } \\
\text { Revista Eletrônica do Curso de Geografia } \\
\text { Graduação e Pós-Graduação } \\
\text { Htpp://www.revistas.ufg.br/geoambiente } \\
\text { Jataí-GO | n } 37 \text { | Mai-Agos/2020 }\end{array}$ & $\begin{array}{l}\text { :GEO } \\
\text { İAMBIENTE } \\
\text { ISSN } 1679-9860\end{array}$ \\
\hline
\end{tabular}

Onde:

$\mathrm{O}_{\mathrm{i}}$ : são os dados observados.

$\mathrm{t}_{\mathrm{i}}$ : são os valores estimados.

\section{Resultados e Discussão}

\subsection{Análise das séries temporais e pré-processamento dos dados}

A partir das séries históricas de vazão, realizou-se a aplicação de Funções de Autocorrelação amostral (FAC) nos dados, a fim de determinar as maiores autocorrelações da série de vazão com ela própria, ou seja, analisou-se as maiores correlações da vazão em outras defasagens (lags) para inserir nos modelos. Dessa forma, definiu-se a autocorrelação estatística dos dados correspondentes a um intervalo de confiança de $95 \%$ para defasagens de 0 a 25, a partir da aplicação da FAC na série histórica de vazão, conforme demonstrado na Figura 3.

Figura 3 - Gráfico da aplicação da Função de Autocorrelação (FAC) para a série de vazão.

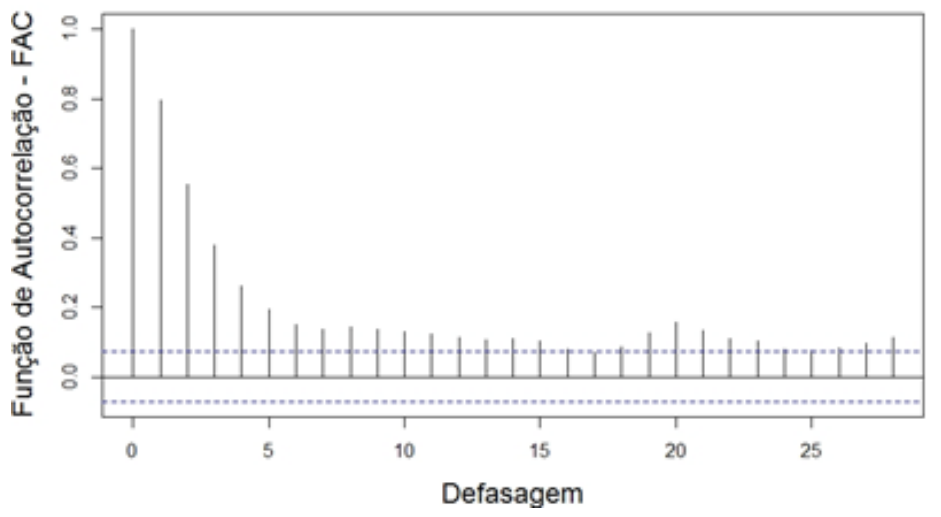

Fonte: Próprio Autores.

Nos correlogramas percebe-se a autocorrelação significativa (nível de significância de 0,05) nas primeiras 5 defasagens da FAC, delimitado pelas linhas tracejadas, o que evidencia uma correlação temporal entre os dados de vazão (V) em diferentes passos (t). Para construção da arquitetura de calibração utilizou-se essas 5 defasagens como variáveis de entrada para calibração dos modelos. 


\begin{tabular}{|c|c|c|}
\hline UFJ/UFG & $\begin{array}{c}\text { GEOAMBIENTE ON-LINE } \\
\text { Revista Eletrônica do Curso de Geografia } \\
\text { Graduação e Pós-Graduação } \\
\text { Htpp://www.revistas.ufg.br/geoambiente } \\
\text { Jataí-GO | n 37 | Mai-Agos/2020 }\end{array}$ & $\begin{array}{l}\text { :GEO } \\
\text { ¿AMBIENTE } \\
\text { ISSN } 1679-9860\end{array}$ \\
\hline
\end{tabular}

A seguir (Figura 4), aplicaram-se Funções de Correlação Cruzada (FCC) entre a série de vazão e as séries de precipitação com o intuito de identificar as melhores defasagens de precipitação pluviométrica para inserir na estrutura de calibração dos modelos a partir dessas séries de dados.

Figura 4 - Gráfico da aplicação da Função de Correlação Cruzada (FCC) entre a série histórica de vazão em contraste com as séries históricas de precipitações dos pluviômetros Glorinha e Anju.
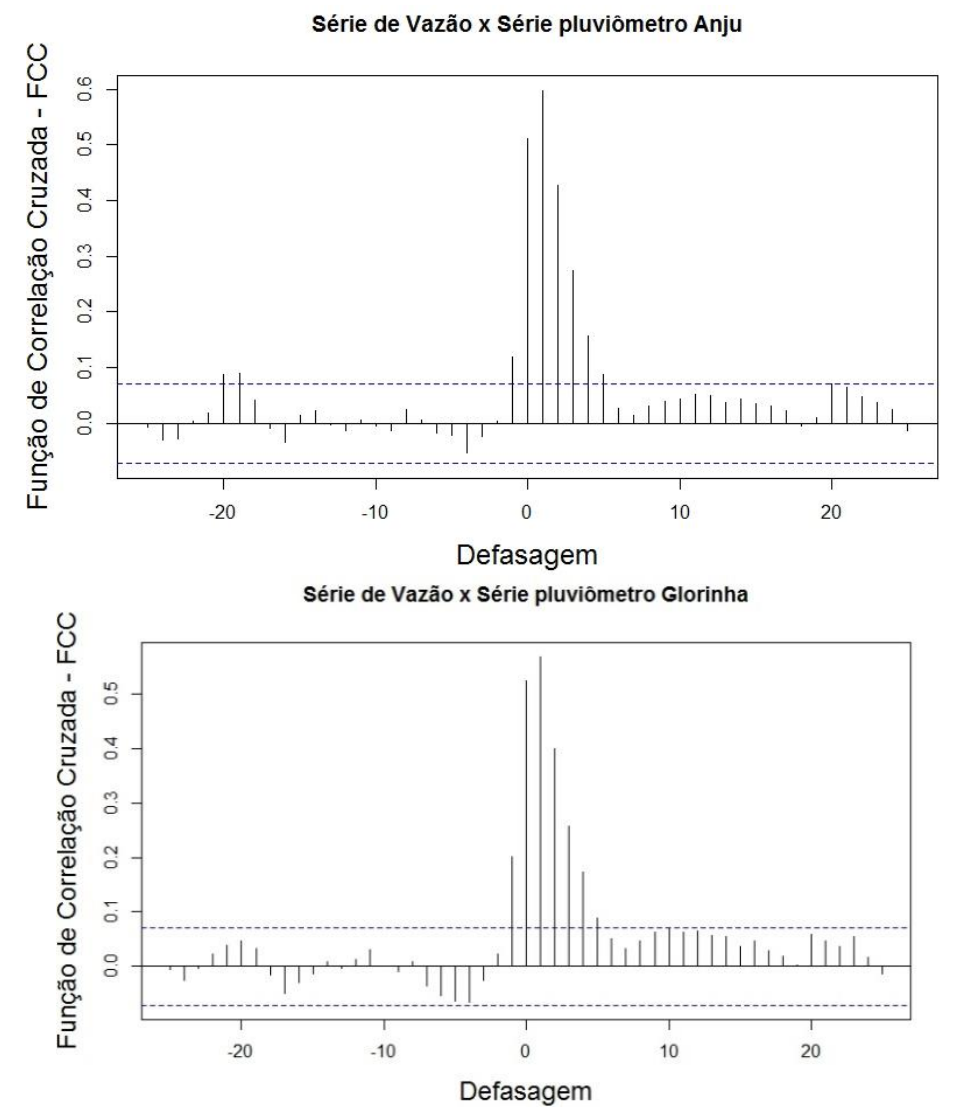

Fonte: Próprio Autores.

Baseado nestas correlações, é possível observar que as defasagens -1, 0, 1, 2, 3, 4 e 5, ou seja, $\mathrm{P}_{\mathrm{t}-1}, \mathrm{P}_{\mathrm{t}}, \mathrm{P}_{\mathrm{t}+1}, \mathrm{P}_{\mathrm{t}+2} \ldots \mathrm{P}_{\mathrm{t}+5}$, considerando a precipitação $(\mathrm{P})$ no tempo $(\mathrm{t})$ foram estatisticamente as mais significativas para os valores medidos da vazão no tempo t. Essa constatação foi observada em ambos os gráficos de correlação da Figura 4.

Neste trabalho, optou-se pela utilização de 5 defasagens negativas de precipitação, usando os valores precipitados dos 5 dias anteriores à medição da vazão $\left(\mathrm{P}_{\mathrm{t}-5}, \mathrm{P}_{\mathrm{t}-4}, \mathrm{P}_{\mathrm{t}-3} \ldots \mathrm{P}_{\mathrm{t}-1}\right)$ para calibração. $\mathrm{O}$ intuito foi criar um modelo autorregressivo para generalização do passo $\mathrm{V}_{\mathrm{t}+1}$. Assim, 


\begin{tabular}{|c|c|c|}
\hline UFJ/UFG & $\begin{array}{c}\text { GEOAMBIENTE ON-LINE } \\
\text { Revista Eletrônica do Curso de Geografia } \\
\text { Graduação e Pós-Graduação } \\
\text { Htpp://www.revistas.ufg.br/geoambiente } \\
\text { Jataí-GO | n 37 | Mai-Agos/2020 }\end{array}$ & $\begin{array}{l}\text { :GEO } \\
\text { :AMBIENTE } \\
\text { ISSN } 1679-9860\end{array}$ \\
\hline
\end{tabular}

foram criadas variáveis que expressam os valores de chuva de cada um dos pluviômetros nas 5 defasagens mencionadas, inserindo maior quantidade de informações para calibração dos modelos.

\subsection{Calibração e análise dos resíduos}

Utilizando os algoritmos discutidos na metodologia, realizou-se a calibração dos modelos utilizando as estruturas observadas na autocorrelação estatística e correlação cruzada das séries. O ajuste dos dados aos algoritmos de aprendizagem foi feito com a precipitação dos dois pluviômetros como variáveis independentes (e suas respectivas defasagens correlacionadas com a vazão) bem como as defasagens autocorrelacionadas da série de vazão. O parâmetro de vazão no tempo $t$ foi utilizada como a variável dependente.

Dessa forma, apresenta-se na tabela 1 os resultados dos estimadores estatísticos (equações 1 e 2) que representam os ajustes dos modelos em ordem quadrática e aritmética.

Tabela 1 - Valores de ajuste para os modelos ANN e GBM para a etapa de calibração.

\begin{tabular}{lll}
\hline Modelo & $\mathbf{R}^{\mathbf{2}}$ & Desvio Absoluto \\
\hline RNA & 0,933 & 397,799 \\
\hline GBM & 0,994 & 108,147 \\
\hline
\end{tabular}

Fonte: Autores, (2020).

A partir da tabela 1 é possível observar os melhores ajustes obtidos. Na referida tabela consta somente a estrutura construída que apresentou os melhores valores do coeficiente de determinação e para o desvio absoluto. Dessa forma é possível notar que ambas metodologias obtiveram bons ajustes para a etapa de calibração para o período avaliado, demonstrando boas capacidades para a generalização dos dados de vazão.

Por meio dos resultados obtidos na etapa de calibração da metodologia de RNA, elaborouse os gráficos de resíduos (vazão observada menos vazão calibrada) juntamente com o histograma de erros (Figura 5) a fim de observar sua dispersão. Foi realizado o teste não paramétrico de 


\begin{tabular}{|c|c|c|}
\hline UFJ/UFG & $\begin{array}{c}\text { GEOAMBIENTE ON-LINE } \\
\text { Revista Eletrônica do Curso de Geografia } \\
\text { Graduação e Pós-Graduação } \\
\text { Htpp://www.revistas.ufg.br/geoambiente } \\
\text { Jataí-GO | n } 37 \text { | Mai-Agos/2020 }\end{array}$ & $\begin{array}{l}\text { :GEO } \\
\text { :AMBIENTE } \\
\text { ISSN } 1679-9860\end{array}$ \\
\hline
\end{tabular}

Kolmogorov-Smirnov (KS) sobre as distribuições das frequências dos erros a fim de verificar sua normalidade.

Figura 5 Gráficos dos resíduos de ajuste e histograma dos erros utilizando RNA.

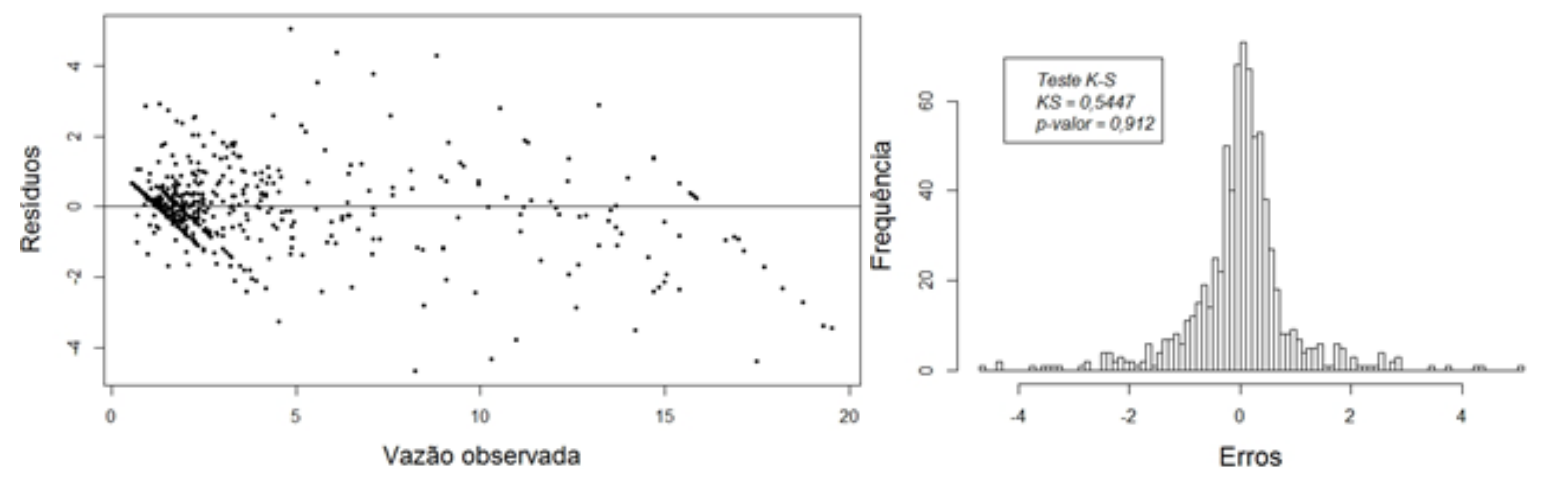

Fonte: Próprio Autores.

Apesar do bom ajuste obtido para a metodologia que utiliza RNA, os gráficos de resíduos obtiveram, visualmente, uma dispersão considerável em torno da linha de resíduo zero com elevada variância. É possível observar no gráfico, maior concentração de pontos na região próxima da vazão média $(3,32 \mathrm{~m} 3 / \mathrm{s})$, indicando que o modelo apresenta maior precisão de calibração para dados que estão em torno destes valores. Dessa forma, este modelo pode apresentar maior instabilidade para realizar generalizações de vazões de pico, principalmente quando muito distantes dos valores da média.

O histograma da Figura 5 apresenta uma distribuição que indica normalidade dos erros tendo em vista que o p-valor é maior que a estatística KS, não havendo, assim, evidência para rejeitar a hipótese de normalidade do conjunto de erros.

Da mesma forma, utilizando a técnica de GBM, elaborou-se os gráficos de resíduos referentes à etapa de calibração juntamente com o histograma dos erros cometidos (Figura 6), objetivando verificar graficamente sua dispersão.

Os gráficos de resíduos da Figura 6 para a vazão estimada e observada evidenciaram uma dispersão menor quando comparadas com os gráficos da Figura 5, tendo maior aderência a linha zero do eixo e com variância constante. Este modelo apresentou maior estabilidade no processo de 


\begin{tabular}{|c|c|c|}
\hline UFJ/UFG & $\begin{array}{c}\text { GEOAMBIENTE ON-LINE } \\
\text { Revista Eletrônica do Curso de Geografia } \\
\text { Graduação e Pós-Graduação } \\
\text { Htpp://www.revistas.ufg.br/geoambiente } \\
\text { Jataí-GO | n } 37 \text { | Mai-Agos/2020 }\end{array}$ & $\begin{array}{l}\text { :GEO } \\
\text { ISAMBIENTE } \\
\text { ISSN } 1679-9860\end{array}$ \\
\hline
\end{tabular}

calibração na medida em que consegue estimar as vazões de pico (vazões superiores a $10 \mathrm{~m}^{3} / \mathrm{s}$ ), demonstrando a ocorrência de poucos outliers.

Figura 6 - Gráficos dos resíduos de ajuste e histograma dos erros utilizando GBM.
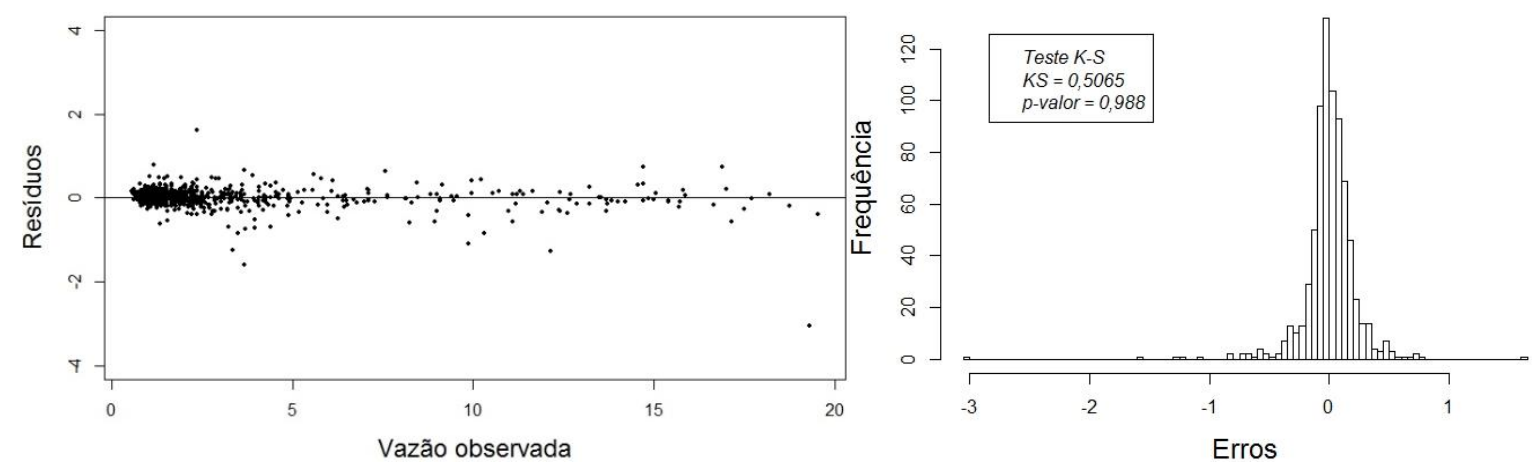

Fonte: Próprio Autores.

O histograma da Figura 6 demonstra baixa incidência de erros cometidos com maior frequência de ocorrência em torno do zero (com alguns poucos outliers no lado negativo do histograma). O teste de normalidade KS para variação dos erros indica uma distribuição normal do conjunto destes dados.

Em geral, ambos modelos apresentaram bom resultados para as métricas de ajuste. Todavia, os modelos calibrados com GBM demonstraram-se mais estáveis e com menores quantidades de outliers. O GBM é uma técnica de ajuste que se baseia na ideia de que encontrar e combinar vários modelos é mais simples do que encontrar um único modelo acurado. A utilização de arquiteturas autorregressivas com várias árvores de regressão em dados de vazão e precipitação demonstraramse mais acuradas para a realização de predições na BHAG.

\section{Conclusões}

- Considerando os valores do coeficiente de determinação e do desvio absoluto calculados, é possível afirmar que ambos os modelos apresentaram bons ajustes de calibração utilizando o método de reamostragem bootstrap. O uso de bootstraping no conjunto de dados demonstrou-se extremamente útil, tendo em vista os poucos dados de monitoramento 


\begin{tabular}{|c|c|c|}
\hline UFJ/UFG & $\begin{array}{c}\text { GEOAMBIENTE ON-LINE } \\
\text { Revista Eletrônica do Curso de Geografia } \\
\text { Graduação e Pós-Graduação } \\
\text { Htpp://www.revistas.ufg.br/geoambiente } \\
\text { Jataí-GO | n 37 | Mai-Agos/2020 }\end{array}$ & $\begin{array}{l}\text { :GEO } \\
\text { ¿AMBIENTE } \\
\text { ISSN } 1679-9860\end{array}$ \\
\hline
\end{tabular}

utilizados para a calibração. O fato da bacia ser pequena (em torno de $120 \mathrm{~km}^{2}$ ) contribuiu para obtenção de modelos com melhor tempo de resposta neste tipo de estrutura.

- A metodologia baseada no algoritmo GBM, apresentou melhor capacidade de calibração aos dados de chuva e de vazão. A partir da análise dos resíduos e frequência dos erros, torna-se evidente que o modelo baseado em GBM, desempenhou performance superior ao modelo baseado em Redes Neurais Artificiais do tipo feedforward na BHAG. O modelo calibrado a partir de GBM apresentou menores erros cometidos e melhores calibrações das vazões de pico, demonstrando ótimo grau de ajuste para este fim.

\section{Referências}

AGÊNCIA NACIONAL DAS ÁGUAS (ANA). Hidroweb - Sistemas de Informações Hidrológicas. Disponível em: <http://www.snirh.gov.br/hidroweb/>. Acesso em: 25 de agosto de 2019.

ALVARES, C. A.; STAPE, J. L.; SENTElHAS, P. C.; DE MORAES, G.; LEONARDO, J.; SPAROVEK, G. Köppen's climate classification map for Brazil. Meteorologische Zeitschrift, v. 22, n. 6, p. 711-728, 2013. Doi: doi.org/10.1127/0941-2948/2013/0507

BARZEGAR, R.; GHASRI, M.; QI, Z.; QUILTY, J.; ADAMOWSKI, J. Using bootstrap ELM and LSSVM models to estimate river ice thickness in the Mackenzie River Basin in the Northwest Territories, Canada. Journal of Hydrology, v. 577, p. 123903, 2019. Doi: doi.org/10.1016/j.jhydrol.2019.06.075

BELLOLI, T. F.; GUASSELLI, L. A. Impactos ambientais decorrentes da orizicultura na APABG. In: GUASSELLI, L Áreas úmidas: questões ambientais. Porto Alegre: UFRGS/Programa de PósGraduação em Geografia. p. 200-234, 2018.

BORRA, S.; DI CIACCIO, A. Measuring the prediction error. A comparison of cross-validation, bootstrap and covariance penalty methods. Computational Statistics and Data Analysis, 54(12), 2976-2989, 2010. Doi: doi.org/10.1016/j.csda.2010.03.004 


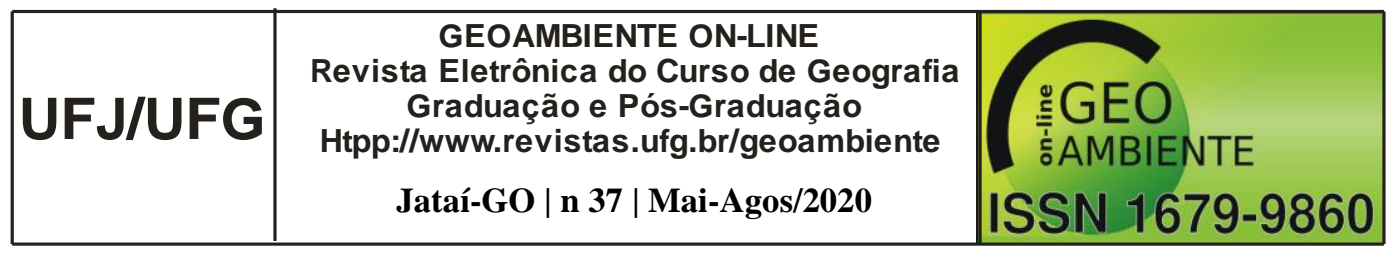

EFRON, B.; TIBSHIRANI, R. Improvements on Cross-Validation: The .632+ Bootstrap Method. JouANNl of the American Statistical Association, v. 92, n. 438, p. 548-560, 1997. Doi: doi.org/10.1080/01621459.1997.10474007

FRIEDMAN, J. H. Greedy function approximation: a gradient boosting machine. The Annals of Statistics, $\quad$ v. 29, n. 5, p. 1189-1232, 2001. Disponível em: < https://www.jstor.org/stable/pdf/2699986.pdf?refreqid=excelsior\%3A02fb611334dbb17b2ccfbb9 $\underline{8 \mathrm{ca} 34 \mathrm{fOce}>}$

FONSECA, T. L.; GORODETSKAYA, Y.; TAVARES, G. G.; DE MELO RIBEIRO, C. B.; DA FONSECA, L. G. A gradient boosting model optimized by a genetic algorithm for short-term riverflow forecast. Revista Mundi Engenharia, Tecnologia e Gestão, v. 4, n. 3, 2019. Doi: http://dx.doi.org/10.21575/25254782rmetg2019vol4n3845

HASTIE, T.; TIBSHIRANI, R.; FRIEDMAN, J. The elements of statistical learning: data mining, inference, and prediction. Springer Science \& Business Media, 2009.

HE, Z.; WEN, X.; LIU, H.; DU, J. A comparative study of artificial neural network, adaptive neuro fuzzy inference system and support vector machine for forecasting river flow in the semiarid mountain region. Journal of Hydrology, v. 509, p. 379-386, 2014. Doi: doi.org/10.1016/j.jhydrol.2013.11.054

KUHN, M. Caret: Classification and Regression Training. Pacote do R versão 6.0. 2019. Disponível em: <https:/CRAN.R-project.org/package=caret>. Acesso em 5 de junho 2019.

LI, C. Y.; LIN, S. S.; LIN, Y. F.; KAN, P. S. A bootstrap regional model for assessing the longterm impacts of climate change on river discharge. International Journal of Hydrology Science and Technology, v. 9, n. 1, p. 84-108, 2019. Doi: doi.org/10.1504/IJHST.2019.096802

LIAO, S.; LIU, Z.; LIU, B.; CHENG, C.; JIN, X.; ZHAO, Z. Multi-step ahead daily inflow forecasting using ERA-Interim reanalysis dataset based on gradient boosting regression trees. Hydrology and Earth System Sciences Discussions, v. 24, p. 2343-2363, 2020. Doi: doi.org/10.5194/hess-24-2343-2020.

KOHAVI, R. A study of cross-validation and bootstrap for accuracy estimation and model selection. In: International Joint Conference on Articial Intelligence. 1995. p. 1137-1145. 


\begin{tabular}{|c|c|c|}
\hline UFJ/UFG & $\begin{array}{c}\text { GEOAMBIENTE ON-LINE } \\
\text { Revista Eletrônica do Curso de Geografia } \\
\text { Graduação e Pós-Graduação } \\
\text { Htpp://www.revistas.ufg.br/geoambiente } \\
\text { Jataí-GO | n 37 | Mai-Agos/2020 }\end{array}$ & $\begin{array}{l}\text { :GEO } \\
\text { İAMBIENTE } \\
\text { ISSN } 1679-9860\end{array}$ \\
\hline
\end{tabular}

MAIER, H. R.; JAIN, A.; DANDY, G. C.; SUDHEER, K. P. Methods used for the development of neural networks for the prediction of water resource variables in river systems: Current status and future directions. Environmental modelling \& software, v. 25, n. 8, p. 891-909, 2010. Doi: doi.org/10.1016/j.envsoft.2010.02.003

MOSAVI, A.; OZTURK, P.; CHAU, K.W. Flood Prediction Using Machine Learning Models: Literature Review. Water, v. 10, n. 11, p. 1536, 2018. Doi: doi.org/10.3390/w10111536

NI, L.; WANG, D.; WU, J.; WANG, Y.; TAO, Y.; ZHANG, J.; LIU, J. Streamflow forecasting using extreme gradient boosting model coupled with Gaussian mixture model. Journal of Hydrology, v. 586, 2020. Doi: doi.org/10.1016/j.jhydrol.2020.124901

PATTENGALE, N. D.; ALIPOUR, M.; BININDA-EMONDS, O. R.; MORET, B. M.; STAMATAKIS, A. How many bootstrap replicates are necessary?. Journal of computational biology, v. 17, n. 3, p. 337-354, 2010.

R CORE TEAM. R: A language and environment for statistical computing. R Foundation for Statistical Computing, Vienna, Austria. Disponível em: 〈http://www.R-project.org>. Acesso em: 13 de janeiro de 2020.

RIDGEWAY, G. Generalized Boosted Models: A guide to the gbm package. 2019. R-Cran, disponível em: 〈https://cran.r-project.org/web/packages/gbm/vignettes/gbm.pdf>. Acesso em 23 de jan. 2019.

RIO GRANDE DO SUL. Decreto $n^{\circ} 38.971$, de 23 de outubro de 1998. Cria a Área de Proteção Ambiental do Banhado Grande, e dá outras providências. Publicado no Diário Oficial do Estado em 26 de outubro de 1998.

RIPLEY, B.; VENABLES, W. net: Feed-forward neural networks and multinomial log-linear modelsR. 2020. R-Cran, disponível em: 〈https://cran.r-project.org/web/packages/nnet/nnet.pdf〉. Acesso em 03 de fev. 2020.

SECRETARIA ESTADUAL DO MEIO AMBIENTE (SEMA). Área de Proteção Ambiental do Banhado Grande. Disponível em:<https://www.sema.rs.gov.br/area-de-protecao-ambiental-dobanhado-grande>. Acesso em 15 de maio de 2020. 


\begin{tabular}{|c|c|c|}
\hline UFJ/UFC & $\begin{array}{c}\text { GEOAMBIENTE ON-LINE } \\
\text { Revista Eletrônica do Curso de Geografia } \\
\text { Graduação e Pós-Graduação } \\
\text { Htpp://www.revistas.ufg.br/geoambiente } \\
\text { Jataí-GO | n } 37 \text { | Mai-Agos/2020 }\end{array}$ & $\begin{array}{l}\text { šGEU } \\
\text { ¿AMBIENTE } \\
\text { ISSN } 1679-9860\end{array}$ \\
\hline
\end{tabular}

SENENT-APARICIO, J.; JIMENO-SÁEZ, P., BUENO-CRESPO, A., PÉREZ-SÁNCHEZ, J., \& PULIDO-VELÁZQUEZ, D. Coupling machine-learning techniques with SWAT model for instantaneous peak flow prediction. Biosystems engineering, v. 177, p. 67-77, 2019. Doi: doi.org/10.1016/j.biosystemseng.2018.04.022

SNELDER, T. H.; LAMOUROUX, N.; LEATHWICK, J. R.; PELLA, H.; SAUQUET, E.; SHANKAR, U. Predictive mapping of the natural flow regimes of France. Journal of Hydrology, v. 373, n. 1-2, p. 57-67, 2009. Doi: doi.org/10.1016/j.jhydrol.2009.04.011

SOUSA, W. S.; DE SOUSA, F. de AS. Rede neural artificial aplicada à previsão de vazão da Bacia Hidrográfica do Rio Piancó. Revista Brasileira de Engenharia Agrícola e Ambiental-Agriambi, v. 14, n. 2, p. 173-180, 2010. Doi: doi.org/10.1590/S1415-43662010000200008

WENGER, S. J.; OLDEN, J. D. Assessing transferability of ecological models: An underappreciated aspect of statistical validation. Methods in Ecology and Evolution, v. 3, n 2, p. 260-267, 2012. Doi: doi.org/10.1111/j.2041-210X.2011.00170.x

WORLAND, S. C.; FARMER, W. H.; KIANG, J. E. Improving predictions of hydrological lowflow indices in ungaged basins using machine learning. Environmental modelling \& software, v. 101, p. 169-182, 2018. Doi: doi.org/10.1016/j.envsoft.2017.12.021

ZHANG, A.; SHI, H.; LI, T.; FU, X. A bootstrap method to estimate the influence of rainfall spatial uncertainty in hydrological simulations. Hydrology and Earth System Sciences Discussions, p. 131, 2017. Doi: doi.org/10.5194/hess-2017-273 